\title{
Síndrome de Prune Belly en una paciente adolescente
}

\author{
Prune Belly syndrome in a female adolescent
}

\author{
Ricardo Hernández-Sarmiento, ${ }^{*,+}$ Juan Camilo Gelvez-Nieto, ${ }^{\ddagger}$ \\ Daniela Vergara-Méndez, ${ }^{\ddagger}$ Gustavo Malo-Rodríguez, ${ }^{\ddagger}$ Ricardo Gastelbondo-Amaya, ${ }^{\ddagger}$ \\ Luz Esthella González-Chaparro, ${ }^{\ddagger}$ Mayerly Prada-Rico ${ }^{\ddagger}$ \\ * Residente de Pediatría, Universidad del Rosario; ${ }^{\ddagger}$ Fundación Cardioinfantil, Bogotá DC, Colombia.
}

\begin{abstract}
RESUMEN
Objetivo: Presentar el caso de una paciente adolescente con síndrome de Prune Belly, el cual se considera una asociación poco frecuente en la población del sexo femenino. Presentación del caso: Mujer de 17 años con diagnóstico de síndrome de Prune Belly desde su nacimiento, con defecto en la musculatura abdominal con piel laxa y arrugada, así como malformación anorrectal alta. Después, se detectaron trastornos de la vía urinaria que le ocasionaron infecciones urinarias de repetición. En la adolescencia se detectó poco desarrollo de genitales externos, útero didelfo y escoliosis toracoabdominal. Por las múltiples malformaciones ha requerido diferentes intervenciones quirúrgicas y hospitalizaciones. Conclusiones: El síndrome de Prune Belly en mujeres representa menos del $5 \%$ de los casos. Es importante conocer los hallazgos clínicos para realizar diagnóstico precoz de las complicaciones asociadas. El manejo debe ser multidisciplinario, y debe incluir la intervención de especialistas en Genética.
\end{abstract}

Palabras clave: Síndrome de Prune Belly, malformación de pared abdominal, trastornos vías urinarias, adolescente, mujeres.

\section{INTRODUCCIÓN}

El síndrome de Prune Belly (SPB) es una enfermedad congénita rara caracterizada por una tríada que consiste en deficiencia de la musculatura de la pared

\begin{abstract}
Objective: To describe the case of a female patient with Prune Belly syndrome, which is rare condition. Case presentation: 17-year-old female patient with a diagnosis of Prune Belly syndrome since birth, with defects of abdominal muscles with loose and wrinkled skin, and anorectal malformation. Later, urinary tract defects were detected that caused recurrent urinary infections. In adolescence, little development of external genitalia, didelphys uterus and thoracoabdominal scoliosis were detected. Conclusions: Prune Belly syndrome in women accounts for less than $5 \%$ of cases. It is important to recognize the clinical findings to make an early diagnosis of the associated complications. Management must be multidisciplinary, which should include the participation of specialists in Genetics.
\end{abstract}

Keywords: Prune Belly syndrome, abdominal wall malformation, urinary tract disorders, adolescent, women.

abdominal, criptorquidia bilateral y anomalías del tracto urinario (hidroureteronefrosis, megauréter). Afecta predominantemente a los varones con una incidencia de 3.4-3.8 por 100,000 nacimientos de hombres vivos en los EEUU; este síndrome se presenta en menos del

\footnotetext{
+Correspondencia: RHS, rhernandezs15@hotmail.com

Conflicto de intereses: Los autores declaran que no tienen.

Citar como: Hernández-Sarmiento R, Gelvez-Nieto JC, Vergara-Méndez D, Malo-Rodríguez G, Gastelbondo-Amaya R, González-Chaparro LE et al. Síndrome de Prune Belly en una paciente adolescente. Rev Mex Pediatr. 2020; 87(6); 227-231. https://dx.doi.org/10.35366/97685
} 
$5 \%$ en mujeres. ${ }^{1}$ Los pacientes con defectos de pared abdominal unilateral, o del sexo femenino se clasifican como síndrome pseudo Prune Belly, o bien, síndrome Prune Belly incompleto o parcial. ${ }^{2,3}$

Frolich reportó el SPB por primera vez en 1839 y en 1950 Eagle y Barret informaron sobre nueve casos y los describieron como "síndrome de Eagle-Barret"; sin embargo, Osler acuñó el término "síndrome de Prune Belly", tomando su nombre de los cambios en la pared abdominal. ${ }^{4,5}$ Existen otros sinónimos, como "síndrome de tríada", "síndrome de abdomen en ciruela pasa" y "síndrome de deficiencia de musculatura abdominal".

En la actualidad, existen múltiples teorías acerca de su patogénesis. Algunas incluyen el origen genético heterogéneo, con la implicación de diversas proteínas de contractilidad muscular visceral o de alteraciones de los receptores muscarínicos colinérgicos $\mathrm{M} 3,{ }^{6,7} \mathrm{o}$ bien, que el modo de herencia está ligado al cromosoma X., ${ }^{8,9}$ Otra propuesta describe que hay alteración en el desarrollo del mesodermo, lo cual explicaría las alteraciones morfológicas a nivel genitourinario, gastrointestinal y de otros sistemas. ${ }^{10}$

El diagnóstico puede realizarse durante la gestación, a través de una ecografía obstétrica con evidencia de contornos irregulares en el abdomen asociado con un aumento de su circunferencia, anomalías del tracto urinario, oligohidramnios, ascitis fetal y uraco permeable. Sin embargo, este síndrome se diagnostica con mayor frecuencia al nacimiento por la particular apariencia de la pared abdominal. ${ }^{11}$ Se han propuesto diferentes opciones de tratamiento de esta compleja condición, que requiere la participación de especialistas con un enfoque interdisciplinario. ${ }^{4}$ Ante la escasa literatura sobre esta condición en pacientes de sexo

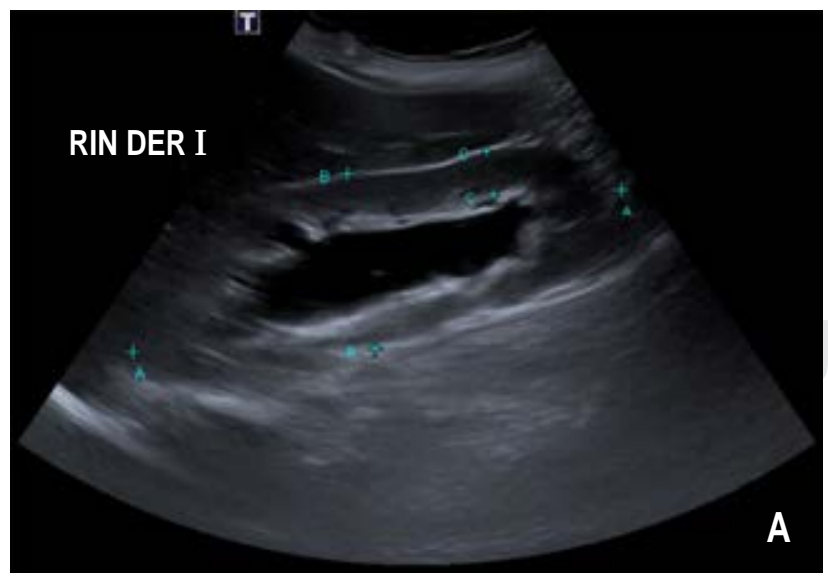

femenino, se presenta el caso de una adolescente con diagnóstico de SPB desde el nacimiento, que ha requerido múltiples intervenciones quirúrgicas y manejo multidisciplinario.

\section{PRESENTACIÓN DEL CASO}

Mujer de 17 años con síndrome de pseudo Prune Belly, quien asiste a manejo en una institución desde su nacimiento. El diagnóstico se realizó en etapa neonatal por la observación de defectos en la musculatura abdominal con piel laxa y arrugada, y después con la identificación en ecografía renal y de vías urinarias de dilatación del tracto urinario superior del lado derecho.

La paciente fue producto del primer embarazo, con controles prenatales adecuados, sin casos similares en la familia. Nació a las 28 semanas de gestación de manera prematura. Ha requerido múltiples hospitalizaciones por procedimientos quirúrgicos e infecciones urinarias. También se ha detectado estreñimiento crónico funcional, escoliosis toracolumbar y discapacidad cognitiva leve.

La primera intervención quirúrgica fue en el periodo neonatal por malformación anorrectal asociada a anomalías del tracto urinario; se realizó vesicostomía, colostomía y anorrectoplastia sagital posterior. La colostomía se cerró a los tres años. A los 12 años requirió realizar vaginoplastia, neovejiga derivada de íleo y colon, apendicovesicostomía (canal Mitrofanoff) por urología pediátrica y cirugía plástica.

Se ha mantenido en vigilancia por nefrología pediátrica por diferentes episodios de infección de vías urinarias (IVU), siendo la última a los 15 años. A los

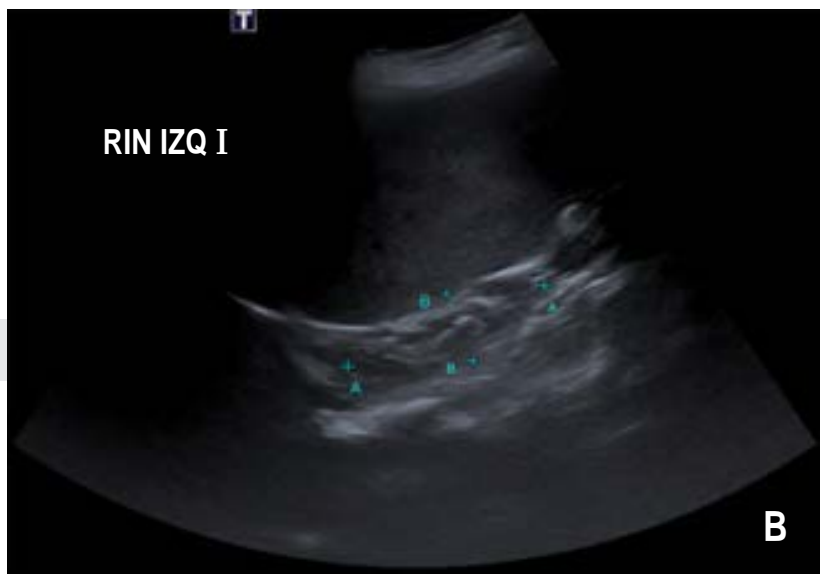

Figura 1: Ecografía renal y de vías urinarias. A) Hidronefrosis del riñón derecho. B) Atrofia renal izquierda. 


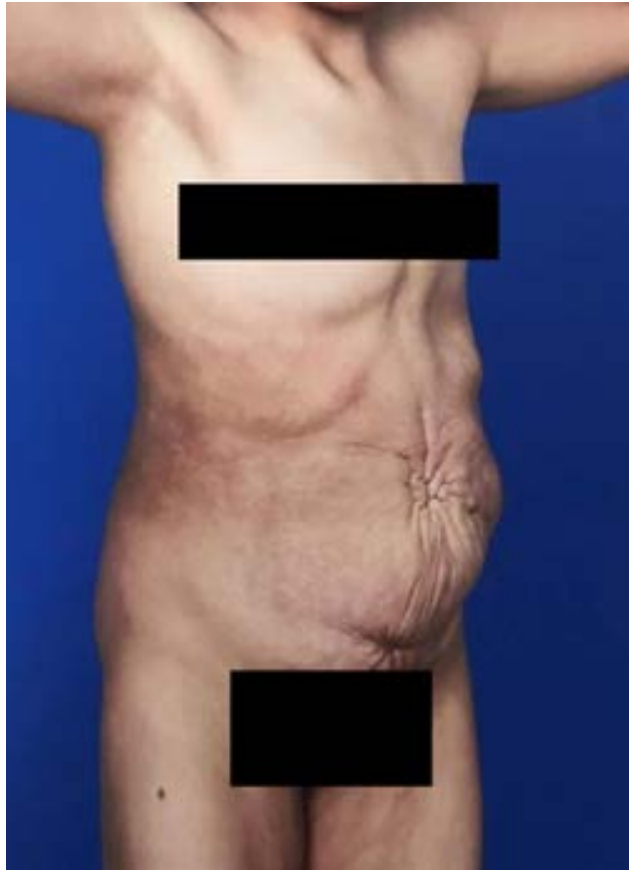

Figura 2: Defecto de pared abdominal.

11 años presentó deterioro de tasa de filtración glomerular (TFG) a $71 \mathrm{~mL} / \mathrm{min} / 1.73$ con gammagrafía renal DMSA, con el cual también se evidenció riñón izquierdo hipoplásico y contribución de 13\% de la TFG. El riñón derecho con hidronefrosis y contribución de 87\%. En último control con ecografía de vías urinarias, se observó hidronefrosis derecha grave (20 mm) (Figura 1A) $\mathrm{y}$ atrofia renal izquierda (Figura 1B).

A la exploración física actual, el abdomen es prominente y globoso, con dibujo de asas intestinales en la piel (Figura 2), y existe escoliosis toracolumbar (Figura 3). Además, se encuentra en seguimiento por ginecología pediátrica porque tiene genitales externos poco desarrollados, útero didelfo (Figura 4) y presenta hemorragia uterina disfuncional. Su manejo es con terapia de reemplazo hormonal y dilataciones vaginales.

Por último, conviene señalar que no se le realizaron estudios moleculares y no fue valorada por el servicio de Genética durante los primeros años de vida.

\section{DISCUSIÓN}

El síndrome de pseudo Prune Belly o Prune-Belly incompleto y el SPB se considera que pertenecen a un solo grupo de defectos congénitos con diferentes grados de afectación y severidad de las manifestaciones.
Sin embargo, a la luz del conocimiento, el término de pseudo Prune Belly se encuentra en desuso.

Las principales anormalidades descritas de esta condición son en la musculatura abdominal, renal y de vías urinarias; sin embargo, entre 65 y $73 \%$ de los casos presentan manifestaciones no urológicas, incluidas anomalías gastrointestinales, ortopédicas, cardiacas, pulmonares y asociaciones con el grupo VACTERL (defectos vertebrales, atresia anal, defectos cardiacos, fístula traqueoesofágica, alteraciones renales y alteraciones en extremidades). ${ }^{12}$ En la presentación en mujeres se describen también malformaciones en genitales.

Las anormalidades renales representan el mayor determinante de supervivencia; alrededor del 30\% de los pacientes presentan IVU o falla renal durante los primeros dos años de vida. ${ }^{13}$ Respecto a las manifestaciones del tracto urinario, a menudo existe dilatación vesical, hidroureteronefrosis y vejiga hipotónica, con escasa capacidad de acomodación por insuficiencia en la contracción del detrusor, lo que ocasiona reflujo vesicoureteral y aumento de residuo postmiccional, el cual es peor en sujetos con válvula uretral posterior, lo que propicia infecciones urinarias y enfermedad renal crónica. ${ }^{1,5,14}$

El SPB tiene una alta morbilidad y mortalidad, de 20 a 30\% mueren en los primeros meses de vida. La mayoría mueren debido a la gran cantidad de alteraciones orgánicas o por las complicaciones inherentes
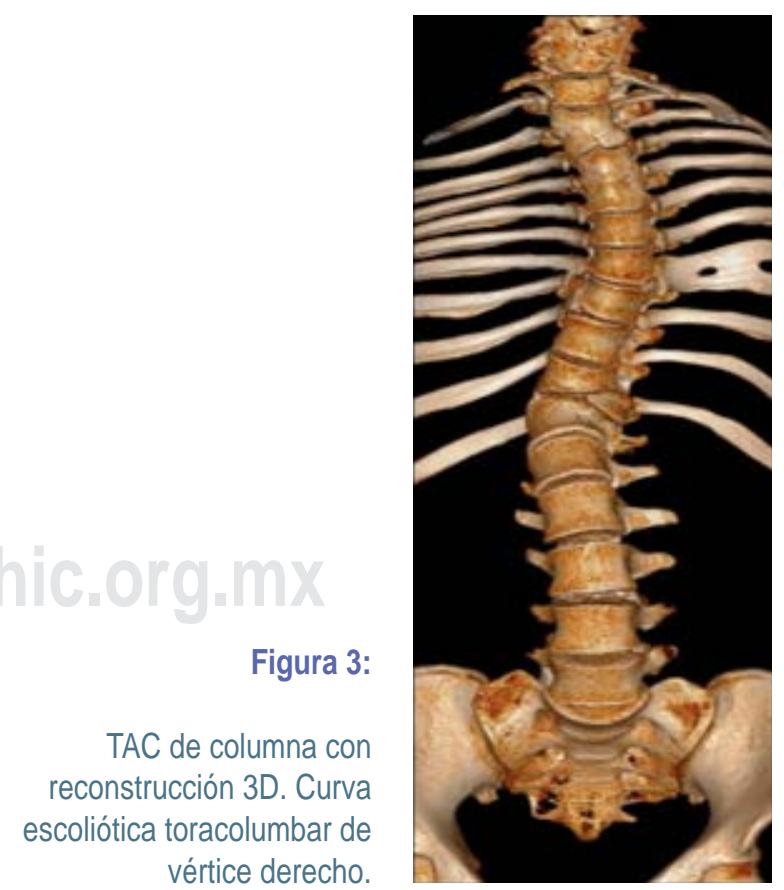


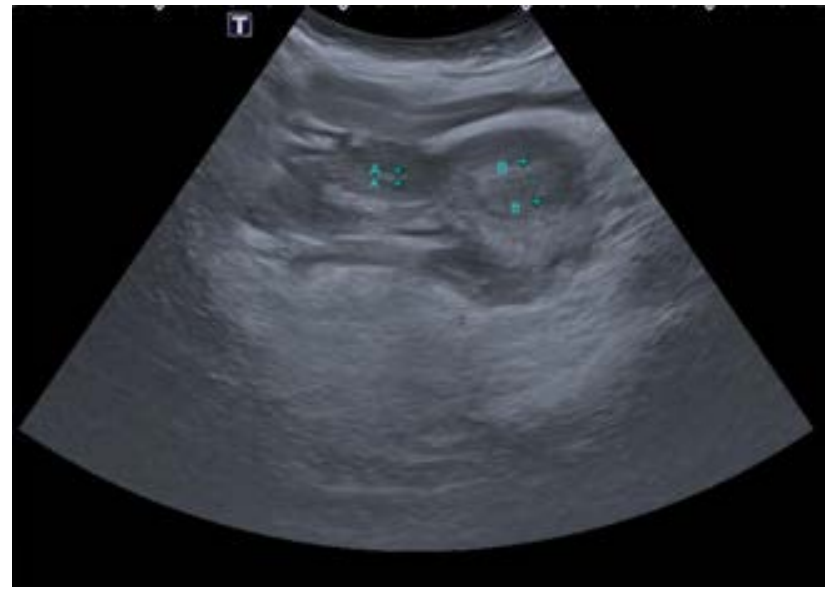

Figura 4: Ecografía pélvica ginecológica transabdominal. Útero didelfo.

a la prematuridad, lo cual está presente en $75 \%$ de los casos. ${ }^{1,15}$ En el caso descrito, presenta las típicas manifestaciones descritas de la pared abdominal, además de anomalías en el tracto urinario y alteraciones ginecológicas (genitales externos subdesarrollados, útero didelfo) malformación anorrectal, así como escoliosis.

Es escasa la literatura científica acerca de mujeres con SPB, en particular de adolescentes o adultas, ya que la mayoría son reportes de casos de recién nacidos, lo que no permite conocer la evolución a largo plazo. Reinberg y colaboradores describieron siete mujeres con SPB y su seguimiento desde el nacimiento, evidenciando falla renal en dos pacientes de las cuatro sobrevivientes, quienes requirieron trasplante renal a los 13 y 14 años respectivamente, de manera exitosa. ${ }^{16}$

Se han considerado múltiples teorías sobre su etiología, siendo las más aceptadas las alteraciones en la embriogénesis como obstrucción al tracto de salida vesical, secundaria a uretra displásica o disfuncional por canalización tardía de la uretra, ${ }^{4,12}$ disgenesia del saco vitelino y trastorno del desarrollo mesodérmico, entre la sexta y décima semana de gestación, lo que explicaría las alteraciones morfológicas en los diferentes órganos y sistemas. ${ }^{3,5,10,12}$ Se han estudiado bases genéticas y mecanismos de herencia para el síndrome y se han propuesto algunos genes implicados en la fisiopatología, como la alteración de los receptores muscarínicos colinérgicos M3 o CHRM3, en el cromosoma 1q43 con herencia autosómica recesiva, el cual favorece el desarrollo del epitelio renal y el músculo de la vejiga. Esto se observó por primera vez en una familia consanguínea con SPB que tenía una mutación homocigótica en dicho gen. ${ }^{7,12,17}$

También se ha estudiado la participación del cromosoma X. En un estudio de Iqbal y colaboradores se identificaron dos pacientes con desequilibrio en el cromosoma X, y uno con duplicación del gen AGTR2 ubicado en dicho cromosoma, el cual está implicado en el desarrollo del tracto urinario. ${ }^{8}$ En otro estudio se identificaron tres mutaciones del gen FLNA, implicado en la producción de la proteína filamina A, la cual regula la reticulación de actina que funciona en las células del músculo liso. ${ }^{9}$ Además, se han propuesto teorías que implican alteraciones en diversas proteínas de contractilidad muscular visceral como resultado de mutaciones genéticas, incluyendo los genes ACTA2, ACTG2, MYH11, MYLK, MYOCD y HNF1B. ${ }^{6}$

El diagnóstico precoz permite un tratamiento oportuno. La descompresión vesical intrauterina se ha descrito mediante la creación de una derivación vesicoamniótica. El diagnóstico prenatal es posible desde las semanas 11 y 14, pero en la mayoría de los casos se realiza en el segundo trimestre. En ultrasonido se observa megavejiga, riñones hiperecoicos, hidrouréteres e hidronefrosis bilateral. ${ }^{4}$ En nuestro caso, el diagnóstico fue en el momento del nacimiento por los hallazgos al examen físico y las alteraciones del tracto urinario evidenciadas por ecografía.

El SPB tiene varias manifestaciones clínicas y una gravedad muy variable, por lo que el tratamiento debe dirigirse a las necesidades específicas del paciente y su calidad de vida. ${ }^{1}$ En la mayoría de los pacientes se realiza injerto de la pared abdominal con una supervivencia del injerto de uno a cinco años de $66.7 \%{ }^{18}$ Los pacientes generalmente se someten a una o dos cirugías por año durante la infancia; sin embargo, no se ha establecido el mejor momento para este procedimiento. Algunos autores proponen hacerlo temprano para evitar la dilatación progresiva del sistema urinario, pero otros argumentan que es preferible un tratamiento conservador inicial. ${ }^{1,4,19}$

\section{REFERENCIAS}

Seidel NE, Arlen AM, Smith EA, Kirsch AJ. Clinical manifestations and management of Prune-Belly syndrome in a large contemporary pediatric population. Urology. 2015; 85(1): 211-215.

2. Jennings RW. Prune Belly syndrome. Semin Pediatr Surg. 2000; 9(3): 115-120.

3. Grover H, Sethi S, Garg J, Ahluwalia AP. Pseudo prune belly syndrome: diagnosis revealed by imaging - a case report and brief review. Pol J Radiol. 2017; 82: 252-257.

4. Hassett S, Smith G, Holland A. Prune belly syndrome. Pediatr Surg Int. 2012; 28(3): 219-228. 
5. Sahin D, Cetiner H, KoC N. The phenotypic and pathological features of Prune-Belly syndrome. Türk Patoloji Dergisi. 2010; 26(2): 114-119.

6. Woolf AS, Lopes FM, Ranjzad P, Roberts NA. Congenital disorders of the human urinary tract: recent insights from genetic and molecular studies. Front Pediatr. 2019; 7: 136.

7. Pachajoa $\mathrm{H}$. De las bases embriológicas a la clínica en el síndrome de Prune Belly. Rev Cubana Obstet Ginecol. 2016; 42(2): 254-259.

8. Iqbal NS, Jascur TA, Harrison S, Chen C, Arevalo MK, Wong D et al. Copy number variations in a population with prune belly syndrome. Am J Med Genet A. 2018; 176(11): 2276-2283.

9. lqbal NS, Jascur TA, Harrison SM, Edwards AB, Smith LT, Choi ES et al. Prune belly syndrome in surviving males can be caused by Hemizygous missense mutations in the X-linked Filamin A gene. BMC Med Genet. 2020; 21(1): 38.

10. Boghossian NS, Sicko RJ, Giannakou A, Dimopoulos A, Caggana $M$, Tsai MY et al. Rare copy number variants identified in prune belly syndrome. Eur J Med Genet. 2018; 61(3): 145-151.

11. Guerrero A, Cuadros C, Archila D, Beltrán S. Síndrome de Prune Belly: presentación de un caso y revisión de la literatura. Salud UIS. 2010; 42(1): 78-85.

12. Tonni G, Ida V, Alessandro V, Bonasoni MP. Prune-belly syndrome: case series and review of the literature regarding early prenatal diagnosis, epidemiology, genetic factors, treatment, and prognosis. Fetal Pediatr Pathol. 2013; 31(1): 13-24.
13. Sutherland RS, Mevorach RA, Kogan BA. The prune-belly syndrome: current insights. Pediatr Nephrol. 1995; 9: 770-778.

14. Garcia M, Grattan J, Arlen A, Smith E, Kirsch A. Detailed evaluation of the upper urinary tract in patients with prune belly syndrome using magnetic resonance urography. J Pediatr Urol. 2016; 12(2): 122.e1-122.e7.

15. Grimsby GM, Harrison SM, Granberg CF, Bernstein IH. Impact and frequency of extra-genitourinary manifestations of prune belly syndrome. J Pediatr Urol. 2015; 11(5): 280.e1-280.e6.

16. Reinberg Y, Shapiro E, Manivel C, Manley C, Pettinato G, González R. Prune belly syndrome in females: A triad of abdominal musculature deficiency and anomalies of the urinary and genital systems. J Pediatr. 1991; 118: 395-398.

17. Weber S, Thiele H, Mir S, Toliat MR, Sozeri B, Reutter $\mathrm{H}$ et al. Muscarinic acetylcholine receptor M3 mutation causes urinary bladder disease and a prune belly-like syndrome. Am J Hum Genet. 2011; 89(5): 668-674.

18. Fusaro F, Zanon G, Ferreli A, Giuliani S, Zacchello G, PasseriniGlazel $\mathrm{G}$ et al. Renal transplantation in prune-belly syndrome. Transpl Int. 2004; 17(9): 549-552.

19. Fischbach M. Ask the expert: is peritoneal dialysis an appropriate technique for small children with prune belly syndrome and terminal renal failure? If yes, how should one perform CAPD or APD in these children? Pediatr Nephrol. 2001; 16: $936-937$ 